\title{
Actividad física en pacientes con diabetes mellitus tipo 2 y relación con características sociodemográficas, clínicas y antropométricas
}

\author{
Physical activity in patients with type 2 diabetes and its relationship with sociodemographic, clinical and \\ anthropometric characteristics
}

Aura Paternina-de la Ossa ${ }^{1}$ orcid.org/0000-0002-1006-2278

Andrés Villaquirán-Hurtado ${ }^{*}$ orcid.org/0000-0002-6156-6425

Sandra Jácome-Velasco ${ }^{1}$ orcid.org//0000-0001-6605-8377

Brigid Galvis-Fernández'1 orcid.org/0000-0001-9065-2835

Yenny Alexandra Granados-Vidal ${ }^{1}$ orcid.org/0000-0002-7177-8958

1 Universidad del Cauca. Popayán, Colombia

Fecha de recepción: Agosto 9 - $2017 \quad$ Fecha de revisión: Noviembre 29 - $2017 \quad$ Fecha de aceptación: Diciembre 21 - 2017

Paternina-de la Ossa A, Villaquirán-Hurtado A, Jácome-Velasco S, Galvis-Fernández B, Granados-Vidal YA. Actividad física en pacientes con diabetes mellitus tipo 2 y relación con características sociodemográficas, clínicas y antropométricas. Univ. Salud. 2018;20(1):72-81. DOI: http://dx.doi.org/10.22267/rus.182001.111

\begin{abstract}
Resumen
Introducción: La Diabetes Mellitus (DM) tipo 2 ha incrementado su prevalencia a nivel mundial, nacional y local, convirtiéndose en una de las principales causas de morbi-mortalidad. Su etiología es múltiple, pero está fuertemente asociada a los hábitos de vida inadecuados tales como los bajos niveles de actividad física y consumo excesivo de azúcares procesados. Objetivo: Determinar el nivel de actividad física en pacientes con diabetes mellitus tipo 2 en la ciudad de Popayán. Materiales y métodos: Se realizó un estudio observacional - descriptivo de 107 participantes, mediante aplicación del cuestionario internacional de actividad física IPAQ y toma de medidas antropométricas como índice cintura-cadera y pliegues cutáneos. Resultados: El 52,3\% de los participantes tenía sobrepeso, el 82,3\% presentó riesgo de comorbilidad y un $36,4 \%$ realizaba actividad física moderada. Se calculó la relación entre las variables índice de masa corporal - cHDL ( $\mathrm{p}=0,029)$, índice cintura cadera (ICC) - presión arterial $(\mathrm{p}=0,266)$, densidad corporal - triglicéridos $(\mathrm{p}=0,278)$, edad e IPAQ $(\mathrm{p}=0,000)$. Conclusión: La diabetes mellitus tipo 2 está asociada a las características antropométricas ligadas al sobrepeso y obesidad, y la falta de actividad física.
\end{abstract}

Palabras clave: Diabetes mellitus tipo 2; actividad física; antropometría; enfermedad crónica; obesidad. (Fuente: DeCS, Bireme).

\begin{abstract}
Introduction: Type 2 Diabetes Mellitus (DM) has increased its prevalence at global, national and local levels, which has become one of the leading causes of morbidity-mortality. Its etiology is multiple, but is strongly associated with inadequate life habits such as low levels of physical activity and excessive consumption of processed sugars. Objective: To determine the level of physical activity in patients with type 2 diabetes mellitus in the city of Popayán. Materials and methods: An observational-descriptive study was conducted with 107 participants, through the application of the international questionnaire of physical activity IPAQ and by taking anthropometric measurements as waist-hip index and cutaneous folds. Results: $52.3 \%$ of participants were overweight, $82.3 \%$ showed a risk of comorbidity and $36.4 \%$ performed moderate physical activity. The relationship among the variables body mass indexHDLC ( $p=0.029$ ), waist hip index (ICC)-blood pressure ( $p=0.266)$, body density-triglycerides $(p=0.278)$, age and IPAQ ( $\mathrm{p}=0.000)$ was calculated. Conclusion: Type 2 diabetes mellitus is associated with anthropometric characteristics linked to overweight and obesity, and lack of physical activity.
\end{abstract}

*Autor de correspondencia

Andrés Villaquirán-Hurtado

e-mail: avillaquiran@unicauca.edu.co 
Keywords: Diabetes mellitus, type 2; exercise; anthropometry; chronic disease; obesity. (Source: DeCS, Bireme).

\section{Introducción}

Según la Federación Internacional de Diabetes (IDF), la Diabetes Mellitus es una enfermedad crónica desencadenada por la pérdida de capacidad para producir células beta pancreáticas secretoras de insulina, dejando así de producir la insulina por reacción autoinmune (Diabetes Mellitus tipo 1 [DM1]) o produciéndola, pero de manera insuficiente (Diabetes Mellitus tipo 2 [DM2]) provocando un aumento en la concentración de glucosa en la sangre(1).

Existe un incremento progresivo e importante de la DM2, convirtiéndose en un problema de salud pública y una de las principales enfermedades crónicas no trasmisibles (ECNT) del siglo; el hombre ha dejado de ser un sujeto activo, dependiente de las nuevas tecnologías de la información y las comunicaciones que lo han llevado a mayor sedentarismo(2-4); así mismo pueden influir factores genéticos, la edad, dietas ricas en grasa y enfermedades como la hipertensión arterial(5). En Colombia se reportan 2.135.380 casos de DM2, con una prevalencia de $8,5 \%$ y de $7,51 \%$ en el caso de la ciudad de Popayán(6,7).

La diabetes ha sido relacionada principalmente con los comportamientos sedentarios de la población; un gasto energético por debajo de 1,5 equivalentes metabólicos (METs) en actividades laborales, en el tiempo de ocio y en las formas de transporte a los sitios de trabajo(8). Comportamiento que no cambia al conocer el padecimiento de Diabetes, Balducci et al., demuestran en su estudio un bajo nivel de actividad física y conductas sedentarias por parte de pacientes con DM2 ${ }^{(9)}$.

La práctica de actividad física juega un rol muy importante en la prevención de enfermedades no trasmisibles y es clave en el control de la glicemia, factores de riesgo cardiometabólicos y complicaciones de la diabetes, así como también permite una mejora en la calidad de vida y bienestar psicológico en pacientes con diabetes ${ }^{(10-}$ 12); 150 minutos semanales de actividad física moderada o vigorosa, como caminar o trotar son recomendadas para pacientes con DM2(13), aunque todavía no hay suficiente claridad sobre la frecuencia, intensidad, tiempo y tipo de ejercicio más favorable para esta enfermedad(14).

Se ha demostrado que el balance energético diario en las personas físicamente activas permite un mejor control de las variables antropométricas como el peso, índice de masa corporal y porcentaje de grasa, efectos que también se ven reflejados en el aumento de la flexibilidad, la velocidad y la fuerza de contracción muscular mediante modificaciones anatómicas y fisiológicas(15-17); además, puede mejorar el perfil lipídico en sangre (triglicéridos, colesterol total y HDL- high density lipoprotein) gracias a una mejor utilización de los lípidos como sustrato energético, disminuyendo la acumulación de grasa visceral y reduciendo el desarrollo de la insulino-resistencia, contribuyendo con la reducción de factores de riesgo cardiovascular(18,19).

En Colombia se han creado normas para la promoción de la actividad física como hábito de vida saludable, por ejemplo la Ley 1122 de 2007 creó el Plan de Salud Pública de Intervenciones Colectivas en el que las entidades territoriales deben elaborar su propio plan para tomar medidas en el control de factores de riesgo para la salud $^{(20)}$ y el Decreto 2771 de 2008 desarrolla y mide el impacto de la actividad física, sin embargo estos esfuerzos no han logrado disminuir la prevalencia de esta patología(21).

Atendiendo lo anterior y con el mínimo conocimiento que hay sobre los niveles de actividad física de los pacientes con diabetes de la ciudad de Popayán, este estudio planteo como objetivo: Determinar el nivel de actividad física en pacientes con DM2. 


\section{Materiales y métodos}

Se realizó un estudio observacional - descriptivo, correlacional, de campo, transversal. Se contactaron 237 pacientes que asistieron a consulta por endocrinología y programas de control de ECNT, en diferentes instituciones prestadoras de servicio de la ciudad de Popayán entre los años 2015 y 2016. Los criterios de inclusión para selección de los pacientes fueron: diagnóstico médico confirmado de DM2, mayor de 18 años, participación voluntaria. Los criterios de exclusión fueron: antecedentes recientes de cirugía, presentar alteraciones cognitivas y/o mentales, retiro voluntario del estudio.

Para calcular el tamaño de muestra se aplicó la técnica de muestreo simple por el programa EPIDAT 3.1 tomando como base el total de la población en la ciudad de Popayán (277.340 habitantes) con el nivel de prevalencia según casos reportados en el año 2014 (7,51\%) y un nivel de confianza del 95\%. El tamaño definitivo de la muestra fue de 107 pacientes con DM2.

Se aplicó una encuesta directa en el domicilio de cada paciente para el registro de características sociodemográficas la cual fue validada y el cuestionario internacional de actividad física (IPAQ) que valora el nivel de actividad física(22). Las características clínicas fueron tomadas de la historia clínica de cada paciente, clasificando cada variable de la siguiente manera: Colesterol total: normal 180 - 200mg/dl; LDL normal < 190 $\mathrm{mg} / \mathrm{dl}$; HDL normal 40-60 mg/dl; VLDL normal 2$30 \mathrm{mg} / \mathrm{dl}(23) ;$ Glucosa en ayunas normal 70-100 $\mathrm{mg} / \mathrm{dl}$; Glucosa aleatoria $<125 \mathrm{mg} / \mathrm{dl}(24)$; Presión arterial normal <119/79(25); IMC normal 18,5 24,9 , sobrepeso 25 - 29,9, obesidad grado I $30-$ 34,9 , grado II $35-39,9$, grado III $>40 \mathrm{~kg} / \mathrm{m} 2$; ICC mujeres normal: 0,71 - 0,84, hombres normal $0,78-0,94(26)$.

Para caracterizar medidas referentes a la situación actual de los pacientes con DM2 se utilizó variables tales como ICC y pliegues cutáneos (densidad corporal), tomadas con una cinta métrica de 1,50 metros $\mathrm{D} \& \mathrm{~T}^{\circledR}$ - DGT ${ }^{\circledR}$ Tape measure y adipómetro Skinfold fat caliper calibrado con un material rígido de medida estándar por una persona capacitada en medidas antropométricas, teniendo como referente las recomendaciones de la International Society for the Advancement of Kinanthropometry (ISAK)(27). Para la obtención del porcentaje de densidad corporal (DC) se aplicó la fórmula: Durnin/Womersley:

$$
D C=C-[M * \log (\text { Suma de los pliegues })]
$$

Posteriormente se determinó el porcentaje de grasa $(\% \mathrm{G})$ mediante la DC aplicando la fórmula:

$$
\% G=\left[\left(\frac{4,95}{D C}\right)-4,5\right] * 100
$$

Donde, la suma de pliegues: bíceps + tríceps + subescapular + suprailíaco del lado derecho, C y M son constantes que dependen de la edad (mayor o igual a 19 años) y el género(28).

Para el análisis de los datos se empleó el programa PSPP y Rwizard software de uso libre. Se realizó un análisis descriptivo a todas las variables y posteriormente un análisis inferencial entre las variables: nivel de actividad física con características sociodemográficas y antropométricas, características clínicas con antropométricas y nivel de actividad física con prueba de correlación Tau b de Kendall. Para todas las pruebas se tomó el nivel de confianza del $95 \%$, significancia con valor de p menor 0,05 y adicionalmente, en las correlaciones, el valor de prueba más cercano a 1 como indicativo de mayor asociación entre las variables. Por tratarse de pruebas para variables ordinales y numéricas no requirieron cumplir requisitos de distribución de normalidad ni homogeneidad de varianzas.

\section{Consideraciones éticas}

El estudio consideró los aspectos éticos establecidos en la Declaración de Helsinki y en la Resolución 008430 de 1993 del Ministerio de Salud y Protección Social de la República de Colombia. Los procedimientos realizados estuvieron dentro de la calificación sin riesgo y se contó con un consentimiento informado debidamente diligenciado con firma y huella de cada uno de los participantes, contemplando el marco de la privacidad y la participación 
voluntaria. El estudio fue avalado por el Comité de Ética del Consejo de Investigaciones de la Universidad del Cauca ${ }^{(29,30) .}$

\section{Resultados}

De los participantes $72 \%(n=107)$ eran de género femenino con un promedio de $66 \pm 11,078$ años, $52,3 \%(n=56)$ de la población se encontraban casados, el $57,9 \%(n=62)$ procedían de la zona urbana, $47,7 \%$ del estrato socioeconómico 3 $44,7 \%(n=51)$. El tiempo de diagnóstico de DM2 tuvo un mínimo de 1 año y un máximo de 50 años,

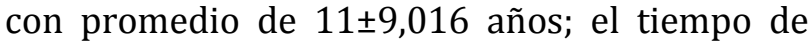
tratamiento de la enfermedad presentó un promedio de 10 $\pm 8,722$ años (Tabla 1).

Tabla 1. Características sociodemográficas PopayánCauca, 2017

\begin{tabular}{|c|c|c|}
\hline Variable & $n$ & $\%$ \\
\hline \multicolumn{3}{|l|}{ Edad } \\
\hline$<50$ & 9 & 8,4 \\
\hline $50-60$ & 19 & 17,8 \\
\hline $61-70$ & 38 & 35,5 \\
\hline $71-80$ & 29 & 27,1 \\
\hline$>80$ & 12 & 11,2 \\
\hline \multicolumn{3}{|l|}{ Género } \\
\hline Masculino & 30 & 28 \\
\hline Femenino & 77 & 72 \\
\hline \multicolumn{3}{|l|}{ Estado civil } \\
\hline Soltero & 40 & 37,4 \\
\hline Casado & 56 & 52,3 \\
\hline Unión libre & 11 & 10,3 \\
\hline \multicolumn{3}{|l|}{ Ocupación } \\
\hline Empleado & 10 & 9,3 \\
\hline Desempleado & 7 & 6,5 \\
\hline Independiente & 17 & 15,9 \\
\hline Pensionado & 27 & 25,2 \\
\hline Ama de casa & 46 & 43 \\
\hline \multicolumn{3}{|c|}{ Zona de procedencia } \\
\hline Urbana & 62 & 57,9 \\
\hline Rural & 45 & 42,1 \\
\hline \multicolumn{3}{|c|}{ Estrato socioeconómico } \\
\hline 1 & 14 & 13,1 \\
\hline 2 & 27 & 25,2 \\
\hline 3 & 51 & 47,7 \\
\hline 4 & 12 & 11,2 \\
\hline 5 & 2 & 1,9 \\
\hline 6 & 1 & 8,9 \\
\hline Total & 107 & 100 \\
\hline
\end{tabular}

Respecto a las características clínicas se obtuvo que un $59,5 \%$ de la población $(n=53)$ presentaron HDL normal, el 33,6\% $(n=36)$ se encontró en nivel óptimo de LDL, 59,8\%, ( $n=64)$ en un nivel deseable de colesterol. El 38,3\% $(n=41)$ de la población tenía elevados los triglicéridos; 57,9\%, $(\mathrm{n}=62)$ de la población mantenían un nivel óptimo de PA (presión arterial) y por último, 62,6\% $(\mathrm{n}=67)$ de los pacientes evaluados tenían la glicemia en ayunas calificada en hiperglucemia (Tabla 2).

Tabla 2. Características clínicas y antropométricas

\begin{tabular}{|c|c|c|}
\hline Variable & $n$ & $\%$ \\
\hline \multicolumn{3}{|l|}{ cHDL } \\
\hline Bajo & 48 & 44,9 \\
\hline Normal & 53 & 59,5 \\
\hline Alto & 6 & 5,6 \\
\hline \multicolumn{3}{|l|}{ cLDL } \\
\hline Óptimo & 36 & 33,6 \\
\hline Límite bajo & 34 & 31,8 \\
\hline Límite alto & 25 & 23,4 \\
\hline Alto & 10 & 9,3 \\
\hline Muy alto & 2 & 1,9 \\
\hline \multicolumn{3}{|l|}{ Colesterol } \\
\hline Deseable & 64 & 59,8 \\
\hline Límite alto & 28 & 26,2 \\
\hline Alto & 15 & 14 \\
\hline \multicolumn{3}{|l|}{ Triglicéridos } \\
\hline Normal & 33 & 30,8 \\
\hline Levemente elevados & 41 & 38,3 \\
\hline Elevados & 31 & 29 \\
\hline Muy elevados & 2 & 1,9 \\
\hline \multicolumn{3}{|l|}{ Presión arterial } \\
\hline Óptimo & 62 & 57,9 \\
\hline Normal & 23 & 21,5 \\
\hline Normal alta & 9 & 8,4 \\
\hline Hipertensión grado I & 13 & 12,1 \\
\hline \multicolumn{3}{|l|}{ Glicemia en ayunas } \\
\hline Hipoglucemia & 1 & 0,9 \\
\hline Normal & 39 & 36,4 \\
\hline Hiperglucemia & 67 & 62,6 \\
\hline \multicolumn{3}{|l|}{ IMC } \\
\hline Bajo peso & 3 & 2,8 \\
\hline Normal & 16 & 15,0 \\
\hline Sobrepeso & 56 & 52,3 \\
\hline Obesidad grado 1 o moderada & 24 & 22,4 \\
\hline Obesidad grado 2 o severa & 7 & 6,5 \\
\hline Obesidad grado 3 o mórbida & 1 & 0,9 \\
\hline \multicolumn{3}{|l|}{ ICC } \\
\hline Sin riesgo de comorbilidad & 18 & 8,6 \\
\hline Con riesgo de comorbilidad & 89 & 83,2 \\
\hline \multicolumn{3}{|l|}{ Densidad Corporal } \\
\hline Excelente & 2 & 1,9 \\
\hline Bueno & 2 & 1,9 \\
\hline Promedio & 6 & 5,6 \\
\hline Pobre & 17 & 15,9 \\
\hline Obeso & 80 & 74,8 \\
\hline
\end{tabular}


Al caracterizar los aspectos antropométricos, se encontró el IMC con una frecuencia mayor en sobrepeso en un porcentaje de $52,3 \%(n=56)$; en las medidas de ICC la frecuencia mayor fue con presencia de riesgo de comorbilidad con un porcentaje de $83,2 \% \quad(n=89)$; en la DC la frecuencia mayor fue obesidad con un porcentaje del 74,8\% (n=80) (Tabla 2).
Por medio de la aplicación de la encuesta IPAQ versión larga, se identificó que el 36,4\% (n=39) de la población presentan un patrón de actividad física moderada, mientras que, de acuerdo a la categorización por dimensiones predomina la intensidad leve en cada uno de ellos, con mayor frecuencia en el trabajo con un porcentaje de 90,7\% (n=97) (Tabla 3).

Tabla 3. Nivel de actividad física de la población participante

\begin{tabular}{|c|c|c|c|c|c|c|c|c|c|c|}
\hline & \multicolumn{8}{|c|}{ IPAQ por dimensiones } & \multicolumn{2}{|c|}{ IPAQ total } \\
\hline & \multicolumn{2}{|c|}{ Trabajo } & \multicolumn{2}{|c|}{ Transporte } & \multicolumn{2}{|c|}{ Hogar } & \multicolumn{2}{|c|}{ Ocio } & \multirow[b]{2}{*}{$n$} & \multirow[b]{2}{*}{$\%$} \\
\hline & $n$ & $\%$ & $n$ & $\%$ & $n$ & $\%$ & $n$ & $\%$ & & \\
\hline Intensidad leve & 55 & 94,8 & 44 & 75,9 & 39 & 67,9 & 41 & 70,7 & 34 & 31,8 \\
\hline Intensidad moderada & 2 & 3,4 & 13 & 22,4 & 15 & 25,9 & 11 & 19 & 39 & 36,4 \\
\hline Intensidad Vigorosa & 1 & 1,7 & 1 & 1,7 & 4 & 6,9 & 6 & 10,3 & 34 & 31,8 \\
\hline Total & & & & & & & & & 107 & 100 \\
\hline
\end{tabular}

Tabla 4. Relación entre nivel de actividad física con características sociodemográficas, clínicas y antropométricas; caracteristicas clínicas con antropométricas

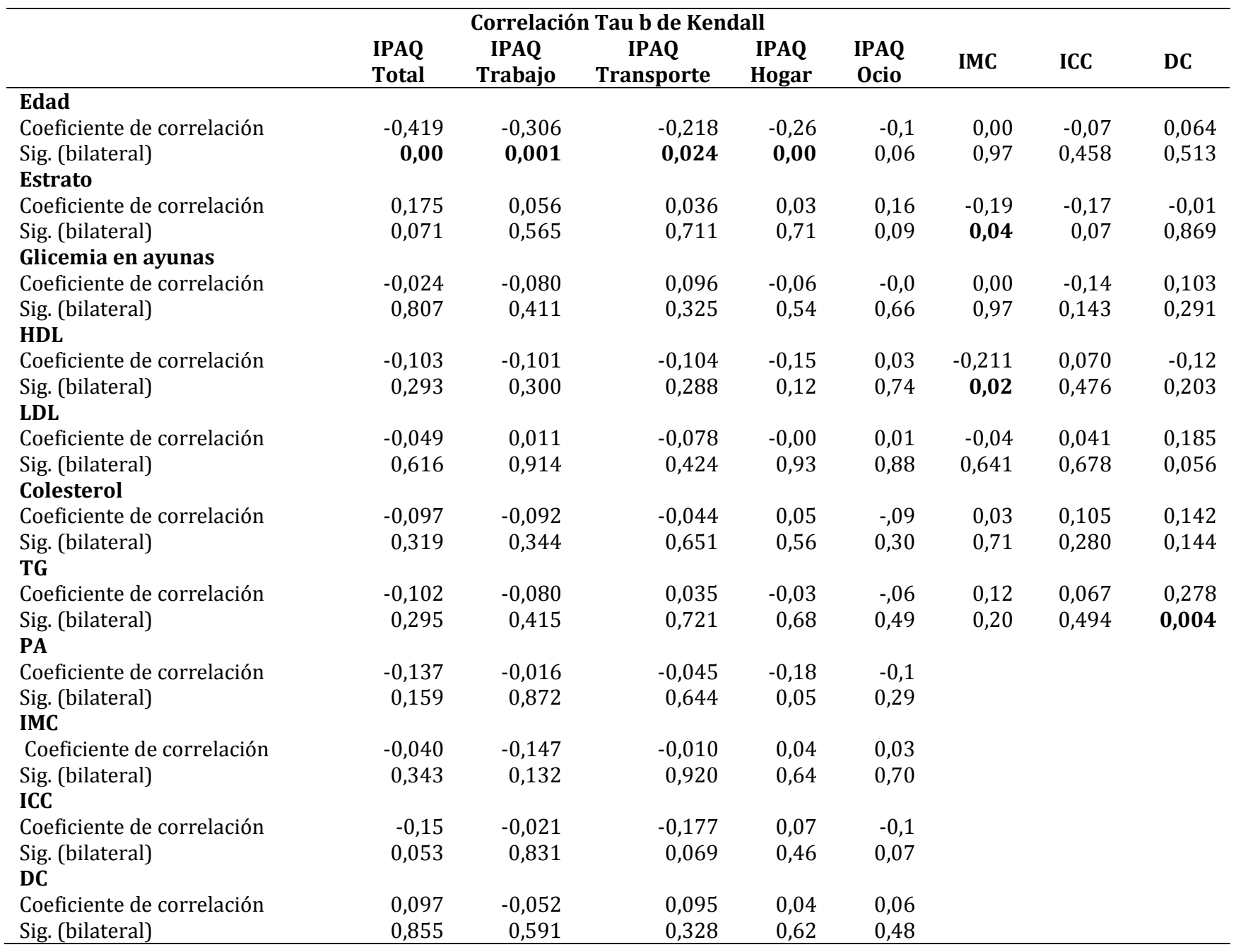


Se encontró relación estadísticamente significativa entre variables sociodemográficas edad con: total $(p=0,000)$, trabajo $(p=0,001)$, transporte $(p=0,024)$, hogar $(p=0,006)$ con una relación inversamente proporcional; y estrato con IMC $(p=0,042)$ (Tabla 4).

Entre las variables antropométricas y clínicas, tales como IMC - HDL se encontró significancia estadística $(p=0,029)$ con una correlación débil inversamente proporcional $(-0,278)$; existe una correlación directamente proporcional débil entre variables como ICC- PA $(0,266)$ y DC-TG $(0,278)$ (Tabla 4$)$.

\section{Discusión}

De acuerdo con las características sociodemográficas, la edad promedio en los pacientes con diabetes del estudio fue de 66 años, investigaciones refieren que la DM2 tiene mayor incidencia en personas de 40 años o más, asociándose a los procesos de envejecimiento y hábitos inadecuados a lo largo de la vida(31). El estado civil casado es un elemento importante para la condición anímica de los adultos; así mismo, los pacientes con ECNT pueden sobrellevar mejor su condición cuando tienen una pareja o un rol definido en su hogar, convirtiéndose en un factor protector frente a las complicaciones de la DM2 ${ }^{(32,33) .}$

Otro factor a tener en cuenta, es la categoría media en el estrato socioeconómico(3) de los pacientes, resultado que difiere con un estudio realizado en la ciudad de Bogotá, donde prevaleció la categoría baja mostrando que la mayoría de los pacientes viven en condiciones de pobreza en países de medianos y bajos ingresos, donde la condición socioeconómica está íntimamente relacionada con la alimentación, salud $y$ alteraciones como obesidad $y$ sobrepeso(34,35).

En el análisis de variables antropométricas cabe resaltar el sobrepeso presentado en los pacientes, varios estudios demuestran la relación entre sobrepeso y obesidad como generadores de afecciones metabólicas que favorecen la aparición de complicaciones en el paciente con DM2(36-38); por esta razón, es importante establecer la distribución de la grasa en el cuerpo, la cual, se asocia con mayor riesgo de enfermedad cardiovascular(39).

Una investigación realizada en la ciudad de Medellín-Colombia con 800 individuos con riesgo cardiovascular, estableció la medición de ICC como marcador de utilidad para la apreciación de alteraciones en la sensibilidad a la insulina; hallando así, semejanzas con este estudio, con presencia de resistencia a la insulina y un porcentaje alto de riesgo en los valores de ICC(40).

Respecto a la medida de IMC es útil para caracterizar el estado nutricional y determinar el grado de sobrepeso u obesidad que tenían los participantes del estudio, pero esta puede ser una medición subjetiva "debido a la deficiente capacidad para distinguir distribución adiposa y/o composición corporal" pudiéndose presentar calificaciones de obeso para personas con un porcentaje de grasa normal(41), pero una masa muscular mayor (riesgo menor de enfermedad coronaria); en comparación con aquellas personas con un IMC normal pero con un exceso de grasa corporal y poco desarrollo de la masa muscular (riesgo potencial de enfermedad coronaria) ${ }^{(36,42-45)}$.

Por otra parte, la medida de los pliegues cutáneos ha sido utilizada en diversas poblaciones, tomando como referencia zonas corporales propensas a acumular tejido adiposo subcutáneo y de esta manera hallar una distribución de la composición corporal más específica (46-48); siendo un método más preciso en la valoración del riesgo cardiovascular(49). Los resultados de esta investigación entre IMC y DC respaldan lo anteriormente expuesto, donde se halló un sobrepeso con la medida del IMC y de obesidad con el método DC.

En contraste a los bajos resultados en el nivel de actividad física de los pacientes con diabetes de la ciudad de Popayán, Manzaneda et al., en un estudio con población peruana, encontraron que más de la mitad de la misma fue clasificada en un nivel de actividad física moderada; lo anterior sugiere que en la comunidad de Popayán es 
necesario fomentar una mayor práctica de actividad física que mejore la condición del paciente y le evite complicaciones ${ }^{(50)}$.

En un estudio piloto realizado en Portugal en el año 2013, se encontró asociación inversamente proporcional entre la edad y la actividad física habitual; resultado similar al presente estudio, donde se halló que a mayor edad, menor nivel de actividad física, aspecto que se debe tener en cuenta por los cambios fisiológicos del proceso de envejecimiento y la alta prevalencia de las ECNT(51-53).

En cuanto a la relación de medidas antropométricas y el perfil lipídico, García et al., en su estudio en la ciudad de Bogotá, obtuvieron que a mayor volumen de grasa visceral, mayores índices aterogénicos con marcadores antropométricos y clínicos más altos ${ }^{(54)}$; resultados muy similares a los encontrados en esta investigación, aunque el procesamiento de los datos para establecerlo no se realizó de igual manera. Otros estudios encontraron relación directamente proporcional entre IMC y LDL como un marcador de arterosclerosis(55).

La asociación entre DC y triglicéridos encontrada en este estudio, se explica con las bases fisiológicas afirman que afirman que la principal función del tejido adiposo es almacenar triglicéridos, por lo cual, al presentarse múltiples alteraciones en la homeostasis energética conlleva a la acumulación de triglicéridos aumentando el tejido graso(56), sin embargo, no hay investigaciones recientes que los correlacione.

Esta investigación estuvo limitada principalmente por el método de recolección de los datos, la extensión de la encuesta y la escasez de estudios enfocados a los factores asociados con la DM2 en el contexto de Popayán. Se espera que esta investigación sirva para futuras exploraciones en programas de intervención en actividad física en la población con diabetes mellitus.

Se sugiere para próximos estudios dividir la muestra en subgrupos, tales como pacientes con diabetes e hipertensión y pacientes con diabetes sin hipertensión, con el fin de establecer un factor diferencial en la respuesta frente al ejercicio, lo cual guiaría de manera más efectiva el tratamiento.

\section{Conclusión}

Las características antropométricas, clínicas y nivel de actividad física basándose en la presencia de sobrepeso y adiposidad, niveles metabólicos aumentados y personas poco activas, pueden aumentar el riesgo para sufrir enfermedades cardiovasculares y complicaciones asociadas a la DM2. Un correcto análisis de la composición corporal del paciente a través de la medición antropométrica, puede ser una herramienta más específica para la valoración del riesgo cardiovascular y la composición corporal, se recomienda continuar considerándola en próximas investigaciones.

El aumento de la prevalencia de ECNT como DM2, se encuentra ligada especialmente al estilo de vida, la composición corporal, la inactividad física de la población y el proceso fisiológico de envejecimiento.

\section{Agradecimientos}

El equipo investigador agradece a los pacientes con diabetes de la ciudad de Popayán, quienes amablemente colaboraron con el estudio, así como a las directivas de las IPS que brindaron información y permitieron realizar este trabajo con sus afiliados. Al programa de Fisioterapia de la Universidad del Cauca por facilitar los mecanismos para la participación de sus estudiantes.

\section{Conflicto de intereses}

Los autores declaramos no tener conflictos de intereses.

\section{Referencias}

1. FID. Diabetes Atlas de la FID [Internet]. Bruselas: FID; 2017. 143 p. Disponible en: www.diabetesatlas.org

2. Chacín M, Rojas J, Pineda C, Rodríguez D, Núñez-Pacheco M, Gómez M, et al. Predisposición humana a la Obesidad, Síndrome Metabólico y Diabetes: El genotipo ahorrador y la incorporación de los diabetogenes al genoma 
humano desde la Antropología Biológica. Diabetes Int. 2011;3(2):36-49.

3. Brown T. "Vulnerability is universal": Considering the place of "security" and "vulnerability" within contemporary global health discourse. Soc Sci Med [Internet]. 2011;72(3):319-26. Disponible en: http://dx.doi.org/10.1016/j.socscimed.2010.09.010

4. Dressler WW. Culture and the risk of disease. Br Med Bull. 2004;69:21-31.

5. Aschner P. Epidemiología de la diabetes en Colombia. Av en Diabetol [Internet]. 2010;26(2):95-100. Disponible en: http://dx.doi.org/10.1016/S1134-3230(10)620054

6. Vargas-Uricoechea H, Casas-Figueroa LÁ. Epidemiología de la diabetes mellitus en Sudamérica: la experiencia de Colombia. Clínica e Investig en Arterioscler [Internet]. 2016;28(5):245-56. Disponible en: http://linkinghub.elsevier.com/retrieve/pii/S0214916 816000176

7. Secretaria de Salud Municipal de Popayán. Análisis de situación de salud con el modelo de determinantes sociales de salud del municipio de Popayán-Cauca. Popayan: Secretaria de Salud; 2014. p. 103-26.

8. Lim MS, Park B, Kong IG, Sim S, Kim SY, Kim J-H, et al. Leisure sedentary time is differentially associated with hypertension, diabetes mellitus, and hyperlipidemia depending on occupation. BMC Public Health [Internet]. 2017;17(1):278. Disponible en: http://bmcpublichealth.biomedcentral.com/articles/1 0.1186/s12889-017-4192-0

9. Balducci S, D’Errico V, Haxhi J, Sacchetti M, Orlando G, Cardelli $\mathrm{P}$, et al. Level and correlates of physical activity and sedentary behavior in patients with type 2 diabetes: A cross-sectional analysis of the Italian Diabetes and Exercise Study_2. PLoS One [Internet]. 2017;12(3):e0173337. Disponible en: http://dx.plos.org/10.1371/journal.pone.0173337

10. Yang Z, Scott CA, Mao C, Tang J, Farmer AJ. Resistance exercise versus aerobic exercise for type 2 diabetes: $A$ systematic review and meta-analysis. Sport Med. 2014;44(4):487-99.

11. Miyamoto T, Fukuda K, Oshima Y, Moritani T. Nonlocomotive physical activity intervention using a triaxial accelerometer reduces sedentary time in type 2 diabetes. Phys Sportsmed [Internet]. 2017;45(3):24551. Disponible en: https://www.tandfonline.com/doi/full/10.1080/0091 3847.2017.1350084

12. Vlaar EMA, Nierkens V, Nicolaou M, Middelkoop BJC, Busschers WB, Stronks $\mathrm{K}$, et al. Effectiveness of a targeted lifestyle intervention in primary care on diet and physical activity among South Asians at risk for diabetes: 2-year results of a randomised controlled trial in the Netherlands. BMJ Open [Internet]. 2017;7:e012221. Disponible en: http://bmjopen.bmj.com/lookup/doi/10.1136/bmjope n-2016-012221

13. Muilwijk M, Stronks K, Qureshi SA, Beune E, CelisMorales C, Gill J, et al. Dietary and physical activity strategies to prevent type 2 diabetes in South Asian adults: protocol for a systematic review. BMJ Open
[Internet]. 2017;7(6):e012783. Disponible en: http://bmjopen.bmj.com/lookup/doi/10.1136/bmjope n-2016-012783

14. McGinley SK, Armstrong MJ, Boulé NG, Sigal RJ. Effects of exercise training using resistance bands on glycaemic control and strength in type 2 diabetes mellitus: a metaanalysis of randomised controlled trials. Acta Diabetol. 2014;52(2):221-30.

15. Serrano-Sanchez, JA, Lera-Navarro A, Espino-Torón L. Physical Activity and Differences of Functional Fitness and Quality of Life in. Int J Med Sci Phys Act Sport. 2013;13(49):87-105.

16. Bernardini A , Manda RM, Burini RC. Características Do Protocolo De Exercícios Físicos Para Atenção Primária Ao Diabetes Tipo 2. Rev Bras Ciência e Mov. 2011;18(3):99-107.

17. Shad BJ, Wallis G, van Loon LJC, Thompson JL. Exercise prescription for the older population: The interactions between physical activity, sedentary time, and adequate nutrition in maintaining musculoskeletal health. Maturitas [Internet]. 2016;93:78-82. Disponible en: http://dx.doi.org/10.1016/j.maturitas.2016.05.016

18. van Dijk JW, Tummers K, Stehouwer C, Hattgens F, van Loon L. Exercise Therapy in Type 2 Diabetes: Is daily exercise required to optimize glycemic control?. Diabetes Care. 2012;35(5):948-54.

19. Duclos M, Oppert JM, Verges B, Coliche V, Gautier JF, Guezennec Y, et al. Physical activity and type 2 diabetes. Recommandations of the SFD (Francophone Diabetes Society) diabetes and physical activity working group. Diabetes Metab [Internet]. 2013;39(3):205-16. Disponible http://dx.doi.org/10.1016/j.diabet.2013.03.005

20. Colombia. Ley Número 1122 de 2007. Bogotá: Congreso de la República; 2007. p. 1-18. Disponible en: http://www.pos.gov.co/Documents/Archivos/Ley 1122 de 2007.pdf

21. Ministerio de Salud y Protección Social. Decreto 2771 de 2008. Bogotá: MinSalud; 2008. p. 1-14. Disponible en: http://www.alcaldiabogota.gov.co/sisjur/normas/Nor ma1.jsp?i=31692

22. Craig CL, Marshall AL, Sjöström M, Bauman AE, Booth ML, Ainsworth BE, et al. International physical activity questionnaire: 12-Country reliability and validity. Med Sci Sports Exerc. 2003;35(8):1381-95.

23. Muntner P, Carey RM, Gidding S, Jones DW, Taler SJ, Wright JT, et al. Potential U.S. Population Impact of the 2017 American College of Cardiology/American Heart Association High Blood Pressure Guideline. Circulation [Internet]. 2017;136(25):1-13 Disponible en: http://circ.ahajournals.org/content/early/2017/11/0 8/CIRCULATIONAHA.117.032582

24. American Diabetes Association. Classification and diagnosis of diabetes. Diabetes Care [Internet]. 2017;40(1):S11-24. Disponible
www.ncbi.nlm.nih.gov/pubmed/27979889.

25. Stone NJ, Robinson JG, Lichtenstein AH, Bairey Merz CN, Blum CB, Eckel RH, et al. 2013 ACC/AHA guideline on the treatment of blood cholesterol to reduce atherosclerotic cardiovascular risk in adults: A report of the American college of cardiology/American heart association task 
force on practice guidelines. J Am Coll Cardiol. 2014;63(25 Part B):2889-934.

26. The Obesity Society. Guidelines for Management of the Obese and Overweight. Obes Res. 1998;6(September):51S-179S.

27. Stewart A, Marfell-Jones M OT. Protocolo Internacional para la Valoración Antropométrica. Sudáfrica: ISAK; 2011.

28. Douglas T, Kennedy J, Quirk E, Yi S, Singh R. Accuracy of Six Anthropometric Skinfold Formulas Versus Air Displacement Plethysmography for Estimating Percent Body Fat in Female Adolescents with Phenylketonuria. JIMD Rep. 2012;4:113-6.

29. Valdespino-Gómez JL, García-García MDL. Declaración de Helsinki. Gac Med Mex. 2001;137(4):391.

30. Ministerio de Salud. Resolución 8430 de 1993. Minist Salud y Protección Soc República Colomb. 1993; 1993(Octubre 4):1-19.

31. Cortés LFL, Ortiz MC, Ruiz AS. Calidad de vida relacionada con la salud en pacientes con diabetes mellitus tipo 2, en un hospital de mediana complejidad en Cali, 2013. Cienc Salud. 2014;2(8):43-8.

32. Suarez Flórez LE, Chavarriaga Ríos MC. Prevalencia de diabetes mellitus autoreportada y factores asociados en los adultos de 60-64 años de Medellín y área metropolitana, participantes en la encuesta nacional de demografía y salud y la encuesta de situación nutricional en Colombia del año 2010. CES Salud Pública. 2014;5(1):21-37.

33. Pérez-Rodríguez A, Maritza D, Gouarnaluses B. Algunos determinantes sociales y su asociación con la diabetes mellitus de tipo 2. Medisan [Internet]. 2015;19(10):3065-8. Disponible en: http://scielo.sld.cu/pdf/san/v19n10/san121910.pdf

34. Álvarez LS, Estrada A, Goez JD, Carreño C, Mancilla L. The effects of socioeconomic status and short stature on overweight, obesity and the risk of metabolic complications in adults. Colomb Med [Internet]. 2013;44(3):146-54. Disponible en: http://www.pubmedcentral.nih.gov/articlerender.fcgi? artid=4002031\&tool=pmcentrez\&rendertype $=$ abstract

35. Pinilla AE, Barrera M del P, Rubio C, Deiva D. Actividades de prevención y factores de riesgo en diabetes mellitus y pie diabético. Acta Medica Colomb [Internet]. 2014;39(3):250-257. Disponible en: http://ezproxy.unal.edu.co/login?url=http://search.eb scohost.com/login.aspx?direct=true $\& \mathrm{db}=$ edssci $\& A N=e$ dssci.S0120.24482014000300008\&lang=es\&site=edslive

36. Zubizarreta-Peinado K, Rodríguez-González N, CastillaRamírez AB, Hevia-Costa M, Becerra-Zayas N. Relation between anthropometric characteristics and its repercussion in Diabetes Type-2. Rev Ciencias Médicas. 2012;16(2):3-19.

37. Mariño-Soler AL, Miguel-Soca PE, Ochoa-Rodríguez M, Cruz-Font JD, Cruz-Batista Y, Rivas-Estévez M. Caracterización clínica, antropométrica y de laboratorio de mujeres con diabetes mellitus tipo 2. Rev Med Electrón. 43(1):57-67.

38. Pérez-Durillo F, Villarejo-Villar AB, Segarra-Robles AB, Ramírez-Sánchez M, Prieto-Gómez I. Pacientes con riesgo cardiovascular elevado en una población de Jaén : antropometría, nutrición y dieta mediterránea. Med fam Andal. 2015;16(1):41-9.

39. Cedeño-Morales R, Castellanos-González M, BenetRodríguez M, Mass-Sosa L, Mora-Hernández C, ParadaArias J. Indicadores antropométricos para determinar la obesidad, y sus relaciones con el riesgo cardiometabólico: cifras alarmantes. Rev Finlay [Internet]. 2015;5(1):12-23. Disponible en: http://scielo.sld.cu/scielo.php?script=sci_arttext\&pid= S2221-

24342015000100003\&lng=es\&nrm=iso\&tlng=es

40. Gallo J, Ochoa J, Balparda J, Aristizábal D. Puntos de corte del perímetro de la cintura para identificar sujetos con resistencia a la insulina en una población colombiana. Acta Med Colomb [Internet]. 2013;38:118-26. Disponible en: http://www.scielo.org.co/pdf/amc/v38n3/v38n3a06. pdf

41. Kweitel S. Bmi : Little Useful Tool To Determine Ideal Weight of a Sportman. J Med Sci Phys Act Sport. 2007;7(28):274-89.

42. Granfeldt-Molina G, Ibarra-Pezo J, Mosso-Corral C, Muñoz-Reyes S, Sáez-Carrillo K, Zapata-Fuentes D. Capacidad predictiva de los índices antropométricos en la detección de Síndrome Metabólico en adultos chilenos. Arch Latinoam Nutr. 2015;65(3):152-7.

43. Fernández-Vázquez R, Romero ÁM, Barbancho MÁ, Alvero-Cruz JR. Predicción del síndrome metabólico por bioimpedancia abdominal y antropometría en hombres de mediana edad. Nutr Hosp. 2015;32(3):1122-30.

44. Bortoletto MSS, Souza RKT de, Cabrera MAS, González AD. Síndrome metabólica, componentes e fatores associados em adultos de 40 anos ou mais de um município da Região Sul do Brasil. Cad Saúde Coletiva [Internet]. 2016;24(1):32-40. Disponible en: http://www.scielo.br/scielo.php?script=sci_arttext\&pi $\mathrm{d}=\mathrm{S} 1414-462 \mathrm{X} 2016000100032 \& \operatorname{lng}=\mathrm{pt} \& \mathrm{tlng}=\mathrm{pt}$

45. Columbié-Llorente Y, Miguel-Soca PE, Rivas-Vázquez D, Borrego-Chi Y. Factores de riesgo asociados con la aparición de diabetes mellitus tipo 2 en personas adultas Risk factors associated to ocurrence of type 2 diabetes mellitus in adults. Rev Cuba Endocrinol. 2016;27(2):123-33.

46. Barretto L, Mackinnon MJ, Poy MS, Wiedemann A, López LB. Estado actual del conocimiento sobre el cuidado nutricional de la mujer embarazada. Rev Española Nutr Humana y Dietética [Internet]. 2014;18(4):226-237. Disponible

en: http://www.renhyd.org/index.php/renhyd/article/vie $\mathrm{w} / 113$

47. Miralles CSW, Wollinger LM, Marin D, Genro JP, Contini V, Dal Bosco SM. Waist-to-height ratio (WHtR) and triglyceride to HDL-c ratio (TG/HDL-c) as predictors of cardiometabolic risk. Nutr Hosp. 2015;31(5):2115-21.

48. Arimura ST, Moura BM, Pimentel GD, Silva MER, Sousa M V. Circunferencia de la cintura es mejor asociado con lipoproteínas de alta densidad (LAD-c) que con el índice de masa corporal (IMC) en adultos con síndrome metabólico. Nutr Hosp. 2011;26(6):1328-32. 
49. Ripka WL, Rotta C V., Ulbricht L, Neves EB. Composición corporal evaluada por pliegues cutáneos y bioimpedancia en varones militares Brasileños. Rev Int Med y Ciencias la Act Fis y del Deport. 2014;14(54):27989.

50. Manzaneda AJ, Lazo-Porras M, Málaga G. Physical activity in outpatients with type 2 diabetes in a national hospital of Peru. Rev Peru Med Exp Salud Pública [Internet]. 2015;32(2):311-5. Disponible en: http://www.scopus.com/inward/record.url?eid=2-

s2.0-

84938873815\&partnerID $=40 \& \mathrm{md} 5=\mathrm{e} 246 \mathrm{e} 744795431$ 68892d446f5ca45dc6

51. Gonçalves ECA, Rinaldi W, Nunes HEG, Capdeboscq MC, Silva DAS. Baixos níveis de atividade física em servidores públicos do sul do Brasil: associac, ão com fatores sociodemográficos, hipercolesterolemia e diabetes. Rev Andal Med Deporte [Internet]. 2010;3(3):87-91. Disponible en: http://www.sciencedirect.com/science/article/pii/S18 88754616300107

52. Mendes R, Días E, Gama A, Castelo-Branco M, ThemudoBarata JL. Prática de exercício físico e níveis de atividade física habitual em doentes com diabetes tipo 2 - estudo piloto em Portugal. Rev Port Endocrinol Diabetes e Metab [Internet]. 2013;8(1):9-15. Disponible en: http://www.sciencedirect.com/science/article/pii/S16 46343913000023\%5Cnhttp://linkinghub.elsevier.com /retrieve/pii/S1646343913000023

53. Salech F, Jara R. Michea L. Cambios fisiológicos asociados al envejecimiento. Rev Med Clin Mondes [Internet]. 2012;23(1):19-29. Disponible en: http://apps.elsevier.es/watermark/ctl_servlet?_f=10\& pident_articulo $=90361755 \&$ pident_usuario $=0 \&$ pcontac tid=\&pident_revista=202\&ty=54\&accion=L\&origen=zo nadelectura\&web $=$ www.elsevier.es\&lan $=$ es\&fichero $=2$ 02v23n01a90361755pdf001.pdf

54. García AI, Niño-Silva L, González-Ruiz K, Ramírez-Vélez R. Volumen de grasa visceral como indicador de obesidad en hombres adultos. Rev Colomb Cardiol. 2016;23(4):313-20.

55. Rojas S, Querales M, Leonardo J, Bastardo P. Nivel de actividad física y factores de riesgo cardiovascular en una comunidad rural del municipio San Diego, Carabobo, Venezuela. Rev Venez Endocrinol Metab [Internet]. 2016;14(2):117-127. Disponible en: http://www.saber.ula.ve/bitstream/123456789/4235 7/1/art3.pdf

56. Gil A. Sánchez F. Tratado de Nutrición: Bases fisiológicas y bioquímicas de la nutrición. 2ed. Madrid: Editorial Médica Panamericana. 2010. 